\title{
EDITORIAL
}

\section{Is the Beneficial Effect of Mediterranean Diet on Cardiovascular Risk Partly Mediated through Better Blood Pressure Control?}

\author{
Panagiotis Anagnostis ${ }^{1}$, George Sfikas ${ }^{2}$, Efthimios Gotsis ${ }^{3}$, Spyridon Karras ${ }^{4}$ and \\ Vasilios G. Athyros,
}

\begin{abstract}
${ }^{I}$ Endocrinology Clinic, Hippocration Hospital, Thessaloniki, Greece; ${ }^{2}$ Department of Internal Medicine, Military Hospital of Thessaloniki, Thessaloniki, Greece; ${ }^{3}$ Second Prop. Department of Internal Medicine, Medical School, Aristotle University of Thessaloniki, Hippokration Hospital, Thessaloniki, Greece; ${ }^{4}$ Department of Endocrinology and Metabolism, Agios Pavlos Hospital, Thessaloniki, Greece
\end{abstract}

Keywords: Mediterranean diet, arterial hypertension, no-pharmacological treatment, cardiovascular disease, healthy diet.

A few days ago, in August 2013, a Cochrane Database Systemic Review reported that the existing limited evidence to date on the effect of Mediterranean diet (MD) on primary prevention of cardiovascular disease (CVD), suggests some favorable effects on risk factors; however, the reduction of CVD event rates was not mentioned [1]. The review included 11 trials (15 papers, 52,044 participants); 7 trials described the intervention as a MD. Clinical events were reported in only one trial [Women's Health Initiative (WHI) 48,835 postmenopausal women with an intervention not described as a MD but increased fruit and vegetable and cereal intake], where no statistically significant effects of the intervention were seen on fatal and non-fatal endpoints during the 8 years of its duration [1]. Since the WHI study was huge and had a great weight in the analysis, it eliminated any other beneficial effect on CVD incidence from other studies. Moreover, in this study the diet used as an intervention was not MD.

On April 2013 the results of the Prevención con Dieta Mediterránea (PREDIMED), a multicenter trial from Spain, were published in New England Journal of Medicine [2]. The study included a total of 7,447 persons (aged 55-80 years) with no CVD at baseline. These were randomized to 1 of 3 diets: a MD supplemented with extra-virgin olive oil, a MD supplemented with mixed nuts or a control diet (advice to reduce dietary fat) [2]. An interim analysis terminated the trial prematurely at 4.8 years. The primary endpoint was the rate of major CVD (myocardial infarction, stroke, or CVD death). The multivariable-adjusted hazard ratios were 0.70 (95\% confidence interval [CI], 0.54 to 0.92$)$ and $0.72(95 \%$ CI, 0.54 to 0.96 ) for the group assigned to a MD with extravirgin olive oil and the group assigned to a MD with nuts,

*Address correspondence to this author at the Department of Internal Medicine, Medical School, Aristotle University of Thessaloniki, Hippokration Hospital, Thessaloniki, Greece. 15 Marmara St, Thessaloniki, 55132, Greece; Tel: +30 2310 892606; Fax: +30 2310 835955;

E-mail: athyros@med.auth.gr or vathyros@gmail.com respectively, vs. the control group [2]. No diet-related adverse effects were reported [2]. Moreover, a post hoc analysis of PREDIMED that will be published in September 2013, in the American Journal of Clinical Nutrition, suggests that one of the mechanisms by which MD, particularly if supplemented with virgin olive oil, can exert health benefits is through changes in the transcriptomic response of genes related to CVD risk [3]. These results of the PREDIMED trial confirm that changes in diet can have beneficial effects on CVD risk. However, the study was criticized for mainly 3 reasons. One is that in MD the dietary pattern as an entity is rather more important than the inclusion or avoidance of specific nutrients [4]. Second there were low (in absolute terms) primary composite CVD outcome rates $(3.8 \%$ and $3.4 \%$ in the intervention groups vs. $4.4 \%$ in the control group) and a minor absolute risk difference (range 0.6 to $1 \%$ ), thus limiting the importance of the study findings [5]. Furthermore there were statistically significant differences in baseline characteristics between the groups: men $(+5.7 \%)$, obese persons $(+4.7 \%)$, diuretic use $(+3.5 \%)$, and oral hypoglycaemic use $(+3.2 \%)$ in the control group than in the intervention group [5]. And third there was a complete lack of policy implications [6]. The PREDIMED trial was neither a pure test of a Mediterranean-style diet nor a pure test of extra-virgin olive oil or nuts. All the above make the interpretation of the PREDIMED trial similarly difficult to that of the Lyon Diet Heart Study [7], which tested provision of a margarine rich in alpha-linolenic acid on top of brief advice to consume a MD in high CVD risk patients with astonishing results [7].

Policymakers [8] and Great Scientific Organizations, like Mayo Clinic [9], already recommend consumption of a Mediterranean-style diet on the basis of a body of evidence from observational and interventional studies, in antithesis to the findings of the Cochrane Database Systemic Review [1]. On the other hand, the policy implications of the PREDIMED trial related primarily to the supplemental foods [2] and not MD itself, thus, we probably have to go both back and elsewhere to find evidence base for the benefits of MD 
and if these are related in any degree to blood pressure (BP) reduction.

The Seven Countries Study showed that the risk and rates of heart attack and stroke both at the population and at the individual level were directly and independently related to the level of serum total cholesterol (TC). It demonstrated that the association between TC level and coronary heart disease (CHD) risk from 5 to 40 years follow-up is found consistently across different cultures $[10,11]$. The Seven Countries Study started in 1958 in former Yugoslavia. In total, 12,763 men, 40-59 years of age, were enrolled as 16 cohorts, in 7 countries, in 4 regions of the world (United States, Northern Europe, Southern Europe, Japan); 1 cohort is in the United States, 2 cohorts in Finland, 1 in the Netherlands, 3 in Italy, 5 in the former Yugoslavia (two in Croatia, and three in Serbia), 2 in Greece ( 1 in Crete and 1 in Corfu), and 2 in Japan [10]. The Seven Countries Study has continued, with high levels of participation, for more than 50 years. The initial and objective data on CVD health in relation to the MD originated from the Seven Countries Study [10]. CHD deaths in the United States and Northern Europe greatly exceeded those in Southern Europe, even when controlled for age, TC and BP levels, smoking, physical activity, and weight [12]. After further investigation, the importance of the eating pattern characterized as the MD became clear $[12,13]$. What exactly is meant by "Mediterranean diet" today, and its benefits, is detailed by other researches during the last 20 years $[14,15]$. During the 90 's, for the first time, the concept of a food pyramid and the need for an adherence to MD score were born [14-16]. As a result of the Seven Countries Study, the MD has been popularized as a "healthy" diet. Nevertheless, it has not replaced the "prudent" diet commonly prescribed to coronary patients [17]. The Crete cohort of the Seven Countries Study had the lowest rates of deaths from CVD [10]; even the 25 year mortality was lower than others (for example vs. the Italian cohort) [18]. This was attributed to the entire lifestyle of Cretans including a variation of MD, the Cretan-type MD. Many investigators during the last 20 years would rather refer to the Cretan-type MD rather than plain MD, which is a rather abstract definition [19-22].

The 40 years' CVD mortality in the Corfu cohort showed that participants also benefited from the long-term adoption of a nutritional pattern (close to the Cretan-type) of MD, the presence of physical activity, optimism, and a positive psychological profile [23]. During the last 15 years, and before the economic crisis, Greeks adopted a more western way of life. Nevertheless, several aspects of the traditional Greek way of life and diet, suggest that a relatively high consumption of vegetables and fruits or olive oil and bread, remained well-established among large segments of the Greek population, and may explain why a population with a few healthy habits still enjoys one of the longer life expectancies among the 16 cohorts of the Seven Country Study 40 years after its initiation [23].

The Working Group on Epidemiology and Prevention of the European Society of Cardiology (ESC) coordinated in 2003 information from 12 European cohort studies, including 205,000 persons, and assessed the 10-year CVD mortality rates. This gave birth to the SCORE (Systematic Coronary Risk Estimation) project [24]. The European Society of
Cardiology encouraged the creation of local SCORES for each country, based on the original SCORE and local data. Indeed the HellenicSCORE (equations and charts) present the calibration of the risk by age group and sex, based on mortality data, as reported by the National Statistical Services of Greece and prevalence data regarding smoking, TC and BP levels, as reported by the ATTICA study [25]. This was very successfully tested in the ATTEMPT Study [26]. The predicated rates of CVD were verified in a survival study with a nearly 4-year follow-up in patients with metabolic syndrome and randomization to intensive versus moderate risk factor treatment [27]. In the meantime the MD score (MedDietScore) was developed, according to the adherence to MD [28]. The weekly consumption of the following 9 food groups: non-refined cereals (whole grain bread and pasta, brown rice, etc.), fruit, vegetables, legumes, potatoes, fish, meat and meat products, poultry, full fat dairy products (like cheese, yoghurt, milk), as well as olive oil and alcohol intake, were included [28]. The inclusion of dietary evaluation (MedDietScore), as well as other sociodemographic and anthropometric characteristics, increases the accuracy and reduces estimating bias of CVD risk prediction models [29]. Thus, we have a country adjusted integrated system that can predict CVD risk. It was clear, during the use of HellenicSCORE and MedDietScore, that a better adherence to MD was related to a lower CVD risk. On the contrary, aging, central fat, hypertension (HTN), diabetes, inflammation, low social status and abstinence from a MD seem to predict CVD events within a 5-year period; actual data from the ATTICA study [30]. All these put the adoption of $\mathrm{MD}$ at a high position among CVD risk factors, for good and for bad, according to the degree of adoption (MedDietScore).

However, does MD affect the level of BP? Is HTN one of the factors to increase CVD risk if the adherence to MD (MedDietScore) is low? There is some evidence on this issue.

It has been suggested by a review on the dietary influences on BP that there is more than enough evidence from observational and clinical studies that diets low in saturated fats and sodium and rich in fruits, vegetables, and fiber, with adequate amounts of potassium, calcium, and magnesium, are effective in the prevention and treatment of HTN alone or as an adjunct to pharmacologic therapy [31]. Such dietary combinations are provided by the MD [31]. The association of adherence to the MD with the incidence of HTN was evaluated among 9,408 men and women enrolled in a dynamic Spanish prospective cohort (SUN) study during 19992005 [32]. The adherence to MD was associated with reduced mean values of systolic BP [moderate adherence, -2.4 $\mathrm{mm} \mathrm{Hg} \mathrm{(95 \%} \mathrm{CI:} \mathrm{-4.0,} \mathrm{-0.8);} \mathrm{high} \mathrm{adherence,}-3.1 \mathrm{~mm} \mathrm{Hg}$ (95\% CI: -5.4, -0.8)] and diastolic BP [moderate adherence, $1.3 \mathrm{~mm} \mathrm{Hg}$ (95\% CI: -2.5, -0.1); high adherence, $-1.9 \mathrm{~mm}$ $\mathrm{Hg}(95 \% \mathrm{CI}:-3.6,-0.1)]$ after 6 years of follow-up [32]. These results suggest that adhering to a Mediterranean-type diet could contribute to the prevention of age-related increase in BP [32]. In the ATTICA Study the mean value of the MedDiet Score was $25.5( \pm 3)$ for men and $27( \pm 3)$ for women $(\mathrm{p}<0.001)$. The prevalence of HTN was $36.6 \%$ in men and $23.7 \%$ in women $(\mathrm{p}<0.001)$ [33]. Diet score was $23.5 \pm 6.4$ in hypertensive and $26.8 \pm 6.6$ in normotensive indi- 
viduals $(\mathrm{p}<0.001)$. The sensitivity of defining people with HTN was higher than for any other CVD risk factor, suggesting that the adoption of MD reduces the risk for HTN [33]. In an elderly population of Cyprus, another Mediterranean Country, $60 \%$ of men and $58 \%$ of women have HTN, along with other classical CVD risk factors [34]. A 10-unit increase in the MedDietScore was associated with $21 \%$ lower odds of having one additional risk factor, including HTN, in women $(\mathrm{p}<0.001)$ and with $14 \%$ lower odds in men $(p=0.05)$ [34]. The results of the CARDIO2000 study [35] point out that the adoption of MD by physically active subjects seems to significantly reduce the coronary heart disease (CHD) events and prevent, just about, the one-third of acute CHD syndromes, in controlled subjects with HTN [35]. This supports the idea that MD combined with physical activity provides substantial protection from acute coronary events in patients with HTN [35]. Other data from the SUN Study also [36] suggest that there is an inverse relationship between fruit and vegetable consumption and the prevalence of nonpreviously diagnosed HTN in a Mediterranean population with a very high intake of both fat (paradoxically) and plantderived foods [36]. There was a $77 \%$ reduction in the prevalence of HTN for those with the higher consumption of both fruit and vegetables compared with those at the lower quintiles of both food groups [36]. This inverse relationship was also evident when considering $\mathrm{BP}$ as a continuous variable, with a mean systolic BP and diastolic BP of $2.2 \mathrm{mmHg}$ lower for those with the highest consumption of fruit and vegetables compared with those with the lowest intake [36]. The study concludes that in a Mediterranean population, with an elevated fat consumption, a high fruit and vegetable intake is inversely associated with BP levels [36]. From the same (SUN) study it was reported that there is an inverse association between fiber or fruit/vegetable consumption and weight gain, thus emphasizing the importance of replacing some dietary compounds by such foods and fiber-rich products, which may help to avoid weight gain [37]. This brinks about the issue of MD and obesity, mainly central, which is the key clinical manifestation of metabolic syndrome (MetS); this includes HTN.

Data suggest that the prevalence of MetS has dramatically increased during the recent years, especially in Western Countries and South East Asia [38]. More than one third (35 $\%$ ) of adults in the U.S. could be characterized as having the MetS, which translates to nearly 84 million U.S. adults affected by MetS [381. Unfortunately the prevalence of MetS in Mediterranean Countries is high (one forth of the adult population), although definitely lower than that in U.S. $[39,40]$. It has been shown by prospective cohort studies, cross-sectional studies and clinical trials that adherence to the MD was associated with reduced risk of the MetS and its individual components also, in particular waist circumference, triglycerides levels, low density lipoprotein cholesterol (HDL-C), BP levels and glucose metabolism [38,41]. These effects of MD increase life expectancy in patients with MetS [42].

On the other hand, Mediterranean countries, such as Greece, have experienced a rapid social-economic change in the last 15 years and recently an economic crisis; both having negative impacts on healthy eating. These community changes affect nutritional habits and there is a tendency to abandon the traditional healthy MD [43]. However, if we continue to try at the elementary school level, things might be better than they look. A study aiming to examine the long-term effects of the "Cretan Health and Nutrition Education Program" on BP, examined several parameters: BP, dietary, anthropometrical and physical activity data nearly 10 years after the original study (at baseline year 1992-1993, and follow-up examination at year 2001-2002) [44]. The findings of the study revealed that the increase over the 10year period in systolic BP and diastolic BP was higher in the control group than in the $\mathrm{MD}$ intervention group $(\mathrm{P}=0.003$ and $\mathrm{P}<0.001$ respectively). These facts are encouraging, indicating favorable changes in BP, micronutrients intake, body mass index (BMI) and physical activity over a 10-year period and 4 years after program's cessation [44]. We just have to keep trying to establish MD at an early age.

In brief, MD reduces CVD risk and this action could be at least in part attributed to the reduction of BP and MetS. Given that the complete adoption of MD is practically impossible, a high degree of adherence is desirable. This could substantially reduce adverse CVD events as well as the incidence of acute coronary syndromes, by one third, If combined with a high level of physical activity. A high adherence to MD also reduces the prevalence of MetS, a part of which is HTN, and diminishes its clinical consequences, improving life expectancy. It is more effective if $\mathrm{MD}$ is adopted early in life.

\section{CONFLICT OF INTEREST}

This editorial was written independently. The authors did not receive financial or professional help with the preparation of the manuscript. The authors have given talks, attended conferences and participated in advisory boards and trials sponsored by various pharmaceutical companies.

\section{ACKNOWLEDGEMENT}

Declared none.

\section{REFERENCES}

[1] Rees K, Hartley L, Flowers N, et al. Mediterranean' dietary pattern for the primary prevention of cardiovascular disease. Cochrane Database Syst Rev 2013; 8: CD009825. [Epub ahead of print]

[2] Estruch R, Ros E, Salas-Salvadó J, et al. PREDIMED Study Investigators. Primary prevention of cardiovascular disease with a Mediterranean diet. N Engl J Med 2013; 368: 1279-90.

[3] Castañer O, Corella D, Covas MI, et al. PREDIMED study investigators. In vivo transcriptomic profile after a Mediterranean diet in high-cardiovascular risk patients: a randomized controlled trial. Am J Clin Nutr 2013; 98: 845-53.

[4] Athyros VG, Kakafika AI, Papageorgiou AA, et al. Effect of a plant stanol ester-containing spread, placebo spread, or Mediterranean diet on estimated cardiovascular risk and lipid, inflammatory and haemostatic factors. Nutr Metab Cardiovasc Dis 2011; 21: 21321.

[5] Estruch R, Ros E, Martínez-González MA. Mediterranean diet for primary prevention of cardiovascular disease. N Engl J Med 2013; 369: 676-7.

[6] Appel LJ, Linda Van Horn L. Did the PREDIMED Trial Test a Mediterranean Diet? N Engl J Med 2013; 368: 1353-4.

[7] de Lorgeril M, Salen P, Martin JL, Monjaud I, Delaye J, Mamelle N. Mediterranean diet, traditional risk factors, and the rate of cardiovascular complications after myocardial infarction: final report of the Lyon Diet Heart Study. Circulation 1999; 99: 779-85. 
[8] Department of Agriculture, Center for Nutrition Policy and Promotion. United States dietary guidelines for Americans, 2010 (http://www.dietaryguidelines.gov).

[9] Mediterranean diet: A heart-healthy eating plan. The heart-healthy Mediterranean is a healthy eating plan based on typical foods and recipes of Mediterranean-style cooking. Here's how to adopt the Mediterranean diet. http://www.mayoclinic.com/health/ mediterranean-diet/CL00011.

[10] Keys A Ed. Seven Countries: A multivariate analysis of death and coronary heart disease. Harvard University Press: Cambridge, Massachusetts 1980 .

[11] Kromhout D. Serum cholesterol in cross-cultural perspective. The Seven Countries Study. Acta Cardiol 1999; 54: 155-8.

[12] Keys A, Aravanis C, Blackburn HW, et al. Epidemiological studies related to coronary heart disease: characteristics of men aged 40-59 in seven countries. Acta Med Scand Suppl 1966; 460: 1-392.

[13] Menotti A, Keys A, Aravanis C, et al. Seven Countries Study. First 20 -year mortality data in 12 cohorts of six countries. Ann Med 1989; $21: 175-9$

[14] Fung TT, Rexrode KM, Mantzoros CS, Manson JE, Willett WC, $\mathrm{Hu} \mathrm{FB}$. Mediterranean diet and incidence of and mortality from coronary heart disease and stroke in women. Circulation 2009; 119 : 1093-100.

[15] Willett WC, Sacks F, Trichopoulou A, et al. Mediterranean diet pyramid: a cultural model for healthy eating. Am J Clin Nutri 1995; 61: $1402 \mathrm{~s}-6 \mathrm{~s}$

[16] Willett WC. Eat, Drink, and Be Healthy: The Harvard Medical School Guide to Healthy Eating. Free Press 2005.

[17] Renaud S, de Lorgeril M, Delaye J, et al. Cretan Mediterranean diet for prevention of coronary heart disease. Am J Clin Nutr 1995; 61(6 Suppl): 1360S-7S.

[18] Dontas AS, Menotti A, Aravanis C, Ioannidis P, Seccareccia F. Comparative total mortality in 25 years in Italian and Greek middle aged rural men. J Epidemiol Community Health 1998; 52: 638-44.

[19] Kafatos A, Verhagen H, Moschandreas J, Apostolaki I, Van Westerop JJ. Mediterranean diet of Crete: foods and nutrient content. J Am Diet Assoc 2000; 100: 1487-93.

[20] Paschos GK, Rallidis LS, Liakos GK, et al. Background diet influences the anti-inflammatory effect of alpha-linolenic acid in dyslipidaemic subjects. Br J Nutr 2004; 92: 649-55.

[21] Simopoulos AP. The Mediterranean diets: What is so special about the diet of Greece? The scientific evidence. J Nutr 2001; 131(11 Suppl): 3065S-73S

[22] de Lorgeril M, Salen P. Modified Cretan Mediterranean diet in the prevention of coronary heart disease and cancer: An update. World Rev Nutr Diet 2007; 97: 1-32.

[23] Panagiotakos DP, Chrysohoou C, Pitsavos C, et el. Forty-years (1961-2001) of all-cause and coronary heart disease mortality and its determinants: the Corfu cohort from the Seven Countries Study. Int J Cardiol 2003; 90: 73-9.

[24] Conroy RM, Pyorala K, Fitzgerald AP, et al. SCORE Project Group. Estimation of ten-year risk of fatal cardiovascular disease in Europe: The SCORE project. Eur Heart J 2000; 24: 987-1003.

[25] Panagiotakos DB, Fitzgerald AP, Pitsavos C, Pipilis A, Graham I, Stefanadis C. Statistical modelling of 10-year fatal cardiovascular disease risk in Greece: the HellenicSCORE (a calibration of the ESC SCORE project). Hellenic J Cardiol 2007; 48: 55-63.

[26] Chimonas T, Athyros VG, Ganotakis E, et al. Assessing the Treatment Effect in Metabolic Syndrome Without Perceptible diabeTes (ATTEMPT) Collaborative Group. Cardiovascular risk factors and estimated 10-year risk of fatal cardiovascular events using various equations in Greeks with metabolic syndrome. Angiology 2010; 61: 49-57.

[27] Athyros VG, Ganotakis E, Kolovou GD, et al. Assessing The Treatment Effect in Metabolic Syndrome Without Perceptible Diabetes (ATTEMPT) Collaborative. Assessing the treatment effect in metabolic syndrome without perceptible diabetes (ATTEMPT): a prospective-randomized study in middle aged men and women. Curr Vasc Pharmacol 2011; 9: 647-57.

[28] Panagiotakos DB, Pitsavos C, Stefanadis C. Dietary patterns: a Mediterranean diet score and its relation to clinical and biological markers of cardiovascular disease risk. Nutr Metab Cardiovasc Dis 2006; 16: 559-68.

[29] Panagiotakos DB, Pitsavos C, Stefanadis C. Inclusion of dietary evaluation in cardiovascular disease risk prediction models increases accuracy and reduces bias of the estimations. Risk Anal 2009; 29: 176-86.

[30] Panagiotakos DB, Pitsavos C, Chrysohoou C, Skoumas I, Stefanadis C; ATTICA Study. Five-year incidence of cardiovascular disease and its predictors in Greece: the ATTICA study. Vasc Med 2008; 13: 113-21.

[31] Kokkinos P, Panagiotakos DB, Polychronopoulos E. Dietary influences on blood pressure: the effect of the Mediterranean diet on the prevalence of hypertension. J Clin Hypertens (Greenwich) 2005; 7: 165-70.

[32] Núñez-Córdoba JM, Valencia-Serrano F, Toledo E, Alonso A, Martínez-González MA. The Mediterranean diet and incidence of hypertension: the Seguimiento Universidad de Navarra (SUN) Study. Am J Epidemiol 2009; 169: 339-46.

[33] Panagiotakos DB, Pitsavos C, Arvaniti F, Stefanadis C. Adherence to the Mediterranean food pattern predicts the prevalence of hypertension, hypercholesterolemia, diabetes and obesity, among healthy adults; the accuracy of the MedDietScore. Prev Med 2007; 44: 33540.

[34] Panagiotakos DB, Polystipioti A, Papairakleous N, Polychronopoulos E. Long-term adoption of a Mediterranean diet is associated with a better health status in elderly people; a cross-sectional survey in Cyprus. Asia Pac J Clin Nutr 2007; 16: 331-7.

[35] Pitsavos C, Panagiotakos DB, Chrysohoou C, et al. The effect of the combination of Mediterranean diet and leisure time physical activity on the risk of developing acute coronary syndromes, in hypertensive subjects. J Hum Hypertens 2002; 16: 517-24.

[36] Alonso A, de la Fuente C, Martín-Arnau AM, de Irala J, Martínez JA, Martínez-González MA. Fruit and vegetable consumption is inversely associated with blood pressure in a Mediterranean population with a high vegetable-fat intake: the Seguimiento Universidad de Navarra (SUN) Study. Br J Nutr 2004; 92: 311-9.

[37] Bes-Rastrollo M, Martínez-González MA, Sánchez-Villegas A, de la Fuente Arrillaga C, Martínez JA. Association of fiber intake and fruit/vegetable consumption with weight gain in a Mediterranean population. Nutrition 2006; 22: 504-11.

[38] Kastorini CM, Panagiotakos DB. The role of the mediterranean diet on the development of the metabolic syndrome. Front Biosci (Elite Ed) 2010; 2: 1320-33.

[39] Athyros VG, Bouloukos VI, Pehlivanidis AN, et al. The prevalence of the metabolic syndrome in Greece: the MetS-Greece Multicentre Study. Diabetes Obes Metab 2005; 7: 397-405.

[40] Anagnostis P. Metabolic syndrome in the Mediterranean region: Current status. Indian J Endocrinol Metab 2012; 16: 72-80.

[41] Kastorini CM, Milionis HJ, Esposito K, Giugliano D, Goudevenos JA, Panagiotakos DB. The effect of Mediterranean diet on metabolic syndrome and its components: a meta-analysis of 50 studies and 534,906 individuals. J Am Coll Cardiol 2011; 57: 1299-313.

[42] Pérez-López FR, Chedraui P, Haya J, Cuadros JL. Effects of the Mediterranean diet on longevity and age-related morbid conditions. Maturitas 2009; 64: 67-79.

[43] Karlén J, Lowert Y, Chatziarsenis M, Fälth-Magnusson K, Faresjö T. Are children from Crete abandoning a Mediterranean diet? Rural Remote Health 2008; 8: 1034.

[44] Kafatos I, Manios Y, Moschandreas J, Kafatos A; Preventive Medicine and Nutrition Clinic University of Crete Research Team. Health and nutrition education program in primary schools of Crete: changes in blood pressure over 10 years. Eur J Clin Nutr 2007; 61: 837-45. 